\title{
HST/STIS Far-UV observations of the central nebulae in the cooling core cluster A 1795
}

\author{
Christopher P. O'Dea ${ }^{1}$, Stefi A. Baum ${ }^{1}$, Jennifer Mack ${ }^{1}$
} Anton M. Koekemoer ${ }^{1}$ and Ari Laor ${ }^{2}$

\author{
${ }^{1}$ Space Telescope Science Institute, 3700 San Martin Dr., Baltimore, MD 21218 USA \\ email: odea@stsci.edu, sbaum@stsci.edu,mack@stsci.edu, koekemoe@stsci.edu \\ ${ }^{2}$ Technion-Israel Institute of Technology, Department of Physics, Haifa, 32000, Israel \\ email: laor@physics.technion.ac.il
}

\begin{abstract}
We present HST/STIS FUV images of the Ly $\alpha$ and FUV continuum emission of the luminous emission line nebulae in the cooling core cluster A1795. The Ly $\alpha$ and FUV continuum emission consist of a diffuse component $(\sim 60 \%)$ and more compact features (knots and filaments) which lie preferentially along the radio source edges. The correlations between the FUV continuum flux and the Ly $\alpha$ emission line flux implies that the nebulae are mainly ionized locally. We suggest that the FUV knots are star clusters with ongoing star formation with rates of several solar masses per year. It appears that star formation occurs through out the nebula, though it is strongly enhanced along the edges of the radio source. The radio source may play a key role in the ionization and morphology of the nebula, e.g., by snow-plowing the gas, shocking and ionizing the gas, triggering star formation, and destroying clouds that it has engulfed. The observed FUV continuum is consistent with about half the number of hot stars which are required to ionize the nebula. We note that we may be missing some of the FUV flux due to obscuration. Thus, we find that young hot stars (e.g., O5) probably provide the bulk of the photons which ionize the nebula, though other sources of ionization may contribute in selected regions of the nebula. We suggest that the mass accretion rates are comparable to the star formation rates (of order $10 M_{\odot} \mathrm{yr}^{-1}$ ). This is consistent with the lack of intermediate temperature gas $(<1 \mathrm{keV})$ being due to energy input to the cooling gas, rather than to "hiding" the cooling gas.
\end{abstract}

\section{Introduction}

The central regions of clusters of galaxies exhibit a rich suite of phenomena, including peaked X-ray surface brightness distributions, short cooling times, radio sources, $\mathrm{cD}$ galaxies, and luminous optical emission line nebula (e.g., Fabian 1994). Although these 'cooling core' clusters have been the subject of intense interest for at least 20 years, they remain an enigma. Recent XMM and Chandra spectral observations of some of the brightest cooling core clusters have only deepened the mystery surrounding these sources. Detections of O VIII demonstrate that some of the central gas is cooler than the surrounding medium, but the lack of commensurate O VII emission suggests that most of the gas never cools below $k T \sim 2 \mathrm{keV}$ (Kaastra et al. 2001; Tamura et al. 2001; Peterson et al. 2001,2003).

We present the first HST/STIS FUV images of the nebulae in the central dominant galaxies in the rich clusters of galaxies A1795. These high resolution data allow us for the first time to investigate the details of the morphology of the Ly $\alpha$ emission line and the FUV continuum. A more detailed discussion of the data analysis and results are given by O'Dea et al. (2004). 


\section{Results}

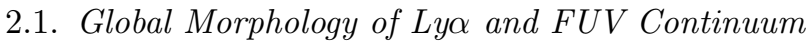

Figure 1 shows a montage of images in several emission lines $(\mathrm{H} \alpha+[\mathrm{NII}], \mathrm{H} \beta+\mathrm{V}$ continuum, and $\operatorname{Ly} \alpha$ ) and the FUV continuum and radio continuum in A1795. The $\mathrm{V}$ and $\mathrm{R}$ band morphologies have been previously described by Pinkney et al. (1996). The relationship between the radio source optical and emission line gas has been discussed by van Breugel, Heckman \& Miley (1984). We see extended FUV continuum and Ly $\alpha$ on the $\sim 20^{\prime \prime}(\sim 20 \mathrm{kpc})$ scale. The Ly $\alpha$ exhibits (1) a diffuse component aligned roughly perpendicular to the overall radio source axis (but roughly 'aligned with the inner radio jet axis), and (2) bright knots which tend to lie along the edges of the radio source; e.g., the knots lie within one kpc of the eastern edge of the southern radio lobe. The FUV continuum is in general similar to the Ly $\alpha$ in morphology, though its large scale axis is more aligned with the large scale axis of the radio source. About $60 \%$ of the Ly $\alpha$ and FUV emission is in the diffuse component, while the rest is in the compact knots.

\subsection{Can Hot Stars Ionize the Nebula?}

Here we consider whether the observed FUV continuum is consistent with a sufficient number of hot stars that they could provide the ionization for the nebula. We assume the most optimistic case where all the ionizing photons from the stars are absorbed by the gas (realistically, some ionizing photons escape thus increasing the number of stars required). We have used the Starburst99 models (Leitherer et al. 1999) to examine whether the observed FUV continuum emission at $1500 \AA$ is consistent with a population of stars which could also produce sufficient ionizing photons to power the nebula. We find that continuous star formation models (a few to few tens of $M_{\odot} \mathrm{yr}^{-1}$ ) can produce the observed FUV continuum and are within a factor of a few of producing the required ionizing photons. This is good agreement considering the existence of significant and variable extinction in the nebula. Thus, our observations are consistent with hot young stars providing at least the bulk of the photons required to ionize the nebula.

If cooling gas is produced at a much higher rate than it is removed by e.g., star formation, a large reservoir of cold gas would accumulate in the inner ICM. The lack of large reservoirs of cold gas in these clusters (e.g., Edge 2001) suggests that the mass accretion rates are fairly modest - of order $10 M_{\odot} \mathrm{yr}^{-1}$. This suggests that the low mass accretion rates inferred by the lack of intermediate temperature gas (e.g., Kaastra et al. 2001; Peterson et al. 2001, 2003) are real. This is consistent with the lack of intermediate temperature gas being due to energy input to the cooling gas, rather than to "hiding" the cooling gas.

We also note that a starburst that produces sufficient ionizing photons to power the nebula would have a bolometric luminosity of $L_{\mathrm{bol}} \sim 10^{44} \mathrm{ergs} \mathrm{s}^{-1}$. We consider the possibility that most of the optical-UV luminosity from the starburst is absorbed by dust and re-radiated in the infrared. If the IR spectral energy distribution is similar to that of the ultra luminous infrared galaxies (Klaas et al. 2001), the flux density at $70 \mu$ would be $S_{70 \mu} \sim 135 \mathrm{mJy}$, which should be easily detectable by SPITZER/MIPS. Thus, SPITZER observations may provide the definitive test of whether hot stars provide sufficient ionizing photons to power the nebula.

\subsection{Energetics of the Starburst}

Here we consider the mechanical energy output from the starburst. The mechanical luminosity from stellar winds and core-collapse supernovae are calculated for the Starburst99 models by Leitherer et al. (1999). Given the estimated star formation rates for a contin- 


\section{A1795}
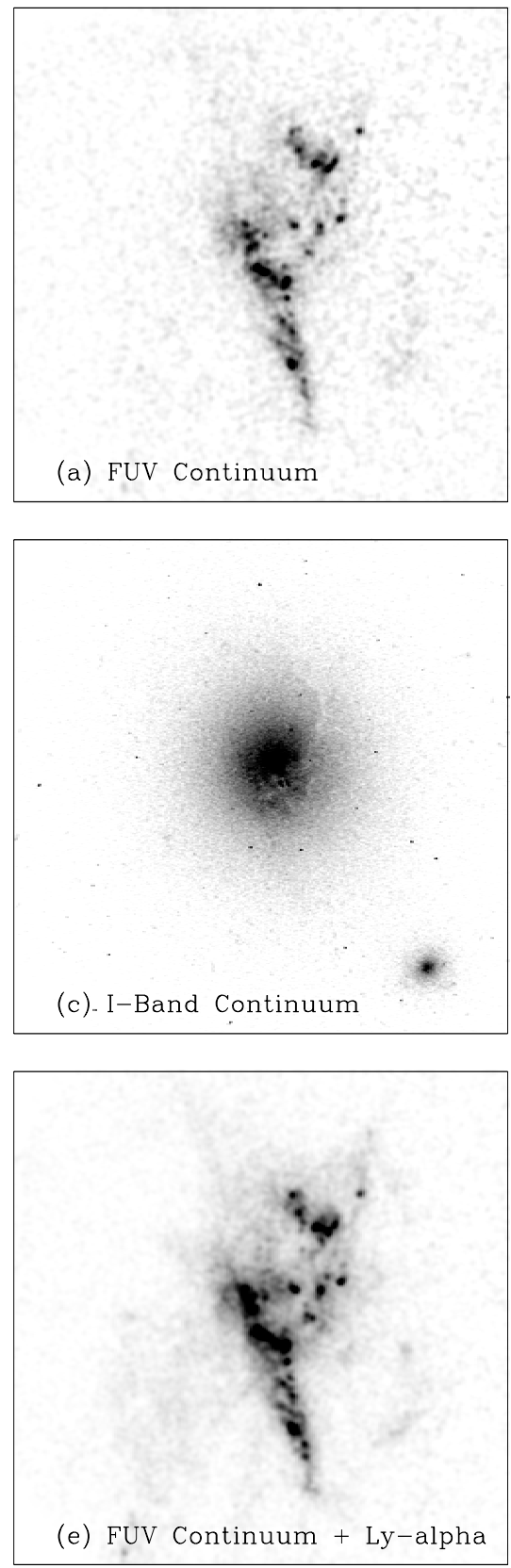

$\left(19^{\prime \prime} \times 19^{\prime \prime}\right)$
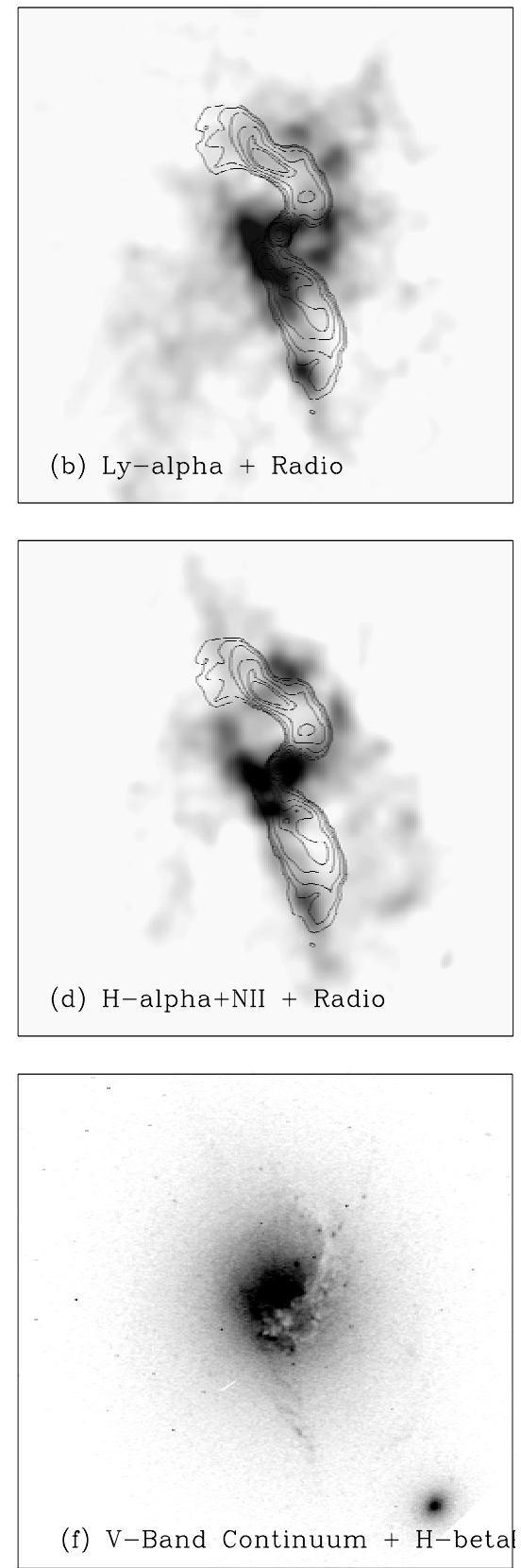

Figure 1. A1795. (J1348+2635). Montage of HST images and VLA $8.4 \mathrm{GHz}$ image. The FUV continuum (a) and Ly $\alpha$ images (b),(e) are from our STIS observations. The other data (c),(d),(f) are archival WFPC2 observations. The Ly $\alpha$ emission-only image (b) is derived from subtracting (a) from (e). The $\mathrm{H} \alpha+[\mathrm{NII}]$ emission image is derived from subtracting the H-band continuum from the WFPC2 F702W image. In order to bring up low surface brightness emission the Ly $\alpha$ and Far-UV continuum images are smoothed with a Gaussian of $\sigma 10$ and 3 pixels, respectively. Note the tendency for the continuum knots and the emission line gas to line along the edges of the radio lobe. VLA image is courtesy of Greg Taylor and Jing Ping Ge (Ge \& Owen 1993). The radio contours are $(0.15,0.20,0.30,0.50,0.80,1.00,2.00,7.00,18.0) \mathrm{mJy} /$ beam. North is up and East is left. The field of view is $19^{\prime \prime} \times 19^{\prime \prime}$. 
uous starburst the expected mechanical luminosity in the range of one to a few $10^{42}$ ergs $\mathrm{s}^{-1}$. Only some fraction of the mechanical energy $(\sim 10 \%)$ will be dissipated as heat the majority will be radiated (e.g., Thornton et al. 1998).

The luminosity radiated by a cooling ICM with a mass accretion rate of $100 M_{\odot} \mathrm{yr}^{-1}$ is $L_{x} \sim 5 \times 10^{43} \mathrm{ergs} \mathrm{s}^{-1}$. The total optical emission line luminosity of the nebulae is similar. Thus, the starburst fails by at least one order of magnitude to provide sufficient mechanical energy to halt the cooling of the hot ICM or to power the luminosity of the emission line nebulae.

\subsection{Snow Plowing by the Radio Source}

We note that there is evidence from the 'bubbles' in the hot X-ray emitting gas in clusters of galaxies that radio sources are able to excavate large cavities in the ICM. In several bubbles the cavities are surrounded by a shell of cooler X-ray emitting gas (e.g., Hydra A - Nulsen et al. 2002; Perseus - Schmidt, Fabian \& Sanders 2002; A2052 - Blanton et al. 2003) and in A2052 there is $\mathrm{H} \alpha$ emission from parts of the X-ray shell (Blanton et al. 2001) indicating that the swept up gas is able to cool and that the expansion of the radio source is not currently highly supersonic.

Thus, we suggest that snow plowing contributes to the formation of arcs of emission line gas along the edges of the radio source. In this scenario, the emission line arcs are due to the diffuse ambient gas which has been swept up by the radio source and compressed and which has subsequently cooled to form cool dense clouds. These clouds should lie just outside the radio source. The velocity of the emission line arc and the expansion velocity of the radio lobe would be similar. Snow plowing is not the only process in the nebula since much of the nebula is not associated with the radio source edges. However, it may contribute to the arcs seen along the radio sources.

\section{Acknowledgements}

Support for program 8107 was provided by NASA through a grant from the Space Telescope Science Institute, which is operate by the Association of Universities for Research in Astronomy, Inc., under NASA contract NAS 5-26555.

\section{References}

Blanton, E. L., Sarazin, C. L., McNamara, B. R., \& Wise, M. W. 2001, ApJ, 558, L15

Blanton, E. L., Sarazin, C. L., \& McNamara, B. R. 2003, ApJ, 585, 227

Edge, A. C. 2001, MNRAS, 328, 762

Fabian, A. C. 1994, ARA\&A, 32, 277

Ge, J. P., \& Owen, F. N. 1993, AJ, 105, 778

Heinz, S., Choi, Yun-Young, Reynolds, C. S., \& Begelman, M. C. 2002, ApJ, 569, 79

Kaastra, J. S., Ferrigno, C., Tamura, T., Paerels, F. B. S., Peterson, J. R., \& Mittaz, J. P. D. 2001, A\&A, 365, 99

Klaas, U., et al. 2001, A\&A, 379, 823

Leitherer, C. et al. 1999, ApJS, 123, 3

Nulsen, P. E. J., David, L. P., McNamara, B. R., Jones, C., Forman, W. R., \& Wise, M. 2002, ApJ, 568, 163

O’Dea, C. P., Baum, S. A., Mack, J., Koekemoer, A., \& Laor, A. 2004, ApJ, in press

Peterson, J. R., et al. 2001, A\&A, 365, 104

Peterson, J. R., et al. 2003, ApJ, 590, 207

Pinkney, J., et al. 1996, ApJL, 468, L13

Tamura, T., et al. 2001, A\&A, 365, L87

Thornton, K., Gaudlitz, M., Janka, H.-Th., \& Steinmetz, M. 1998, ApJ, 500, 95

van Breugel, W., Heckman, T., \& Miley, G. 1984, ApJ, 276, 79 